\title{
LA UTOPÍA DE MORO Y LA ISLA GRANDE DE TIERRA DEL FUEGO, ¿UNA EQUIVALENCIA POSIBLE?
}

MAURICIO ONETTO PAVEZ*

\section{RESUMEN}

La Utopía de Tomas Moro fue publicada en el año 1516. Desde aquel entonces se han realizado una cantidad importante de estudios en torno a lo que representó políticamente, socialmente y económicamente esta obra. Sin embargo, poco se ha reflexionado sobre las dimensiones "reales" que llevaron a Moro a realizar este escrito y sobre las geografías en las que se inspiró para darle vida a su isla de Utopía. Nuestro trabajo propone examinar y analizar cómo se habría forjado esto último, específicamente, el caso de los territorios que inspiraron al autor. Para ello, exhibiremos la gran influencia que tuvieron las anotaciones realizadas por Américo Vespucio en el pensamiento del inglés, sobre todo, aquellas que narró durante sus viajes al extremo sur del continente americano. Una de las hipótesis que se intentará demostrar, será que Moro leyó las cartas de este navegante en donde expuso que su expedición habría estado en zonas cercanas a los grados $52^{\circ}-53^{\circ}$ latitud sur, y que gracias a esta lectura tuvo un punto de referencia para describir y definir los espacios geográficos de la isla de Utopía. En otras palabras, nuestra propuesta sugiere que existirian ciertas equivalencias geográficas destacadas por Moro entre aquella isla imaginaría con las formas que tiene la Isla grande de Tierra del Fuego y sus alrededores. Asimismo, nos interesa introducir sobre las posibles conexiones que pudieron haberse establecido entre los imaginarios y sensibilidades germinados desde este relato con aquellos que circularon sobre el territorio chileno durante el siglo XVI.

PALABRAS CLAVES: Isla, Utopía, Tierra del Fuego, Tomás Moro, Américo Vespucio.

MORO'S UTOPIA AND THE ISLA GRANDE DE TIERRA DEL FUEGO, A POSSIBLE EQUIVALENCE?

\section{ABSTRACT}

Thomas Morus' Utopia was published in 1516. Since then, research about this work has mainly focused on its political, social and economic significance. However, there has been little reflection on the real dimensions that encouraged Morus to create this piece of writing and on the geography that inspired him to bring the Utopia Island to life. Our study intends to shed light on

Doctor (PH.D.) en Histoire et Civilisations, de l'École des Hautes Études en Sciences Sociales (EHESS, París). Profesor Universidad SEK, Facultad Patrimonio Cultural y Educación. maonetto@gmail.com 
the relationship between Morus's work and the landscapes that inspired him. For this purpose, we will analyze the great influence that Americo Vespucio's writings had over Morus' thought. We focus, more specifically, on those pieces penned by Vespucio during his trips to the far south of the American continent. One of the hypotheses we will attempt to prove is that Morus read the navigator's letters, where it is stated that Vespucio went beyond the 52nd-53rd latitude south. Morus would have used Vespucio's letter as a reference to describe and define the geography of Utopia Island. In other words, we suggest that there are certain geographical equivalences between the imagined island and the shape of Tierra del Fuego Island and its surroundings. By the same token, we are interested in exploring possible connections which may have been established between the imaginaries and sensibilities stemming from Morus' text and those which circulated in the Chilean territory during the 16th century.

\section{KEYWORDS: Island, Utopia, Tierra del Fuego, Thomas More, Amerigo Vespucci.}

Tomás Moro, político e intelectual inglés, publicó en 1516 en la ciudad de Lovaina su célebre escrito titulado La Utopía. Desde aquel entonces, se han utilizado los significados $y$ nombre de este relato para explicar múltiples problemáticas sociales y políticas en las distintas épocas que sucedieron a la obra. Ciertamente, existe una exuberante bibliografía sobre la obra $y$ sus usos, pero que en un breve artículo como este, nos es imposible reseñar ${ }^{1}$.

Si tuviéramos que hacer un resumen del escrito de Moro, se podría decir lo siguiente: el texto narra lo que muchos podrían considerar como el desarrollo idílico de una sociedad organizada, un desarrollo basado en el control absoluto de todos los aspectos de la vida social como también aquellos vinculados a la naturaleza. El trabajo, la vida social, la religión y la moral son presentados en una armonía "perfecta" en la sociedad utopiana, aunque tensionados por reglas bastante severas en caso de incumplimientos de una de ellas. Todo esto se experimenta en una isla cuya ubicación es una incógnita ${ }^{2}$. Es por ello que se puede aseverar que La Utopía ha sobresalido y ha sido leída, principalmente, desde un punto de vista cívico-político. Para estudiosos como Louis Marin La Utopía fue ante todo un discurso que buscó

1 Algunos textos que tuvimos en cuenta para realizar este estudio fueron: (Goodey, 1970; Bradshaw, 1981; Shephard, 1976; Duhamel, 1963, Elias, 2014; Heiserman 1963, Raitiere, 1963).

2 Los personajes de la obra -Moro y Gilles- precisan en la primera edición que Utopía significa "lugar de felicidad", que no se encuentra "en ninguna parte". Esta última fue la segunda traducción que hizo el propio Moro. La Utopía,

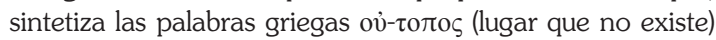

criticar las ideologías y realidades de la sociedad europea de inicios del siglo XVI, por medio de una proposición que ponía en valor lo incognito y sus posibles maravillas- como contraparte a los males que esta presentaba (Marin, 2009, p. 251).

A pesar de lo anterior, creemos que el texto de Moro no sólo respondió a los modos de organización que tenía la sociedad europea de la época, sino que también a lo que se pensaba era el espacio exterior: el Nuevo Mundo o las nuevas fronteras que fueron descubriendo en gran parte los ibéricos (Marin, 1993). En otras palabras, Moro intentó responder no sólo a las injusticias sociales del período, a la "necedad" constante de esos años como decía su amigo Erasmo ${ }^{3}$, sino que también a las curiosidades exteriores que llegaban a las cortes y que no se sabía aún cómo poder representarlas.

El ejercicio de Moro pudo ser posible, porque poseía una gran curiosidad, aunque también por los cargos de poder que tuvo que ejercer. Estos últimos le permitieron ser un observador al día de lo que pasaba en el resto de las cortes y lugares del mundo. Moro fue testigo de las primeras cartas, imágenes y escritos que describían los nuevos descubrimientos, como también al Nuevo Mundo. Todos estos eventos

y $\varepsilon \tilde{v}^{-} \tau$ o $\pi$ os (lugar de felicidad). De ahí que se estructure en torno a ella una representación de la realidad de forma idealizada y sin imperfecciones. Para más detalles, ver el prefacio hecho por Simone Goyard-Fabre sobre este texto (Goyard-Fabre, 1987).

3 Moro dedicó en parte su trabajo a su amigo Erasmo y utilizó ciertos datos de su aclamado texto "Elogio de la locura" o "necedad" como también podía haber sido traducido para esos años (Rotterdam, 1511). 
los aterrizó y los vinculó a los problemas políticos y sociales de la época tanto en su país (reino) como los que vivía el resto de Europa. En este sentido, Moro fue un verdadero "hombre moderno", ya que supo vislumbrar las velocidades de los eventos a una escala mundial ${ }^{4}$.

\section{Un viajero perdido en el Nuevo Mundo}

El libro estuvo compuesto por dos partes (Libro I y II). Todo esto se experimenta en una isla cuya ubicación es una incógnita. Las acciones de la narración cobraron vida a través de un diálogo entre tres hombres, los únicos personajes que componen la obra. Sólo uno de ellos tuvo la experiencia de haber estado en la Isla de Utopía. Esto último se describe detalladamente, principalmente, en el libro II.

Raphael Hythloday se llamó el protagonista de la historia de Moro. Los otros dos personajes, fueron el propio inglés y su amigo Peter Gilles. Según el texto, el protagonista era un navegante reconocido en Europa para esos años. En efecto, para Gilles, Hythloday no era cualquier marinero, sino uno de esos pocos que habían tenido la posibilidad de recorrer el mundo entero. Gilles señalaba que los viajes de este trotamundos podían ser comparados con los de "Ulises o Platón", y que estos le habían otorgado una sabiduría que lo transformaba en un verdadero filósofo (More, 1842, p. 50) 5 .

Hythloday explicitó que había acompañado a Américo Vespucio en sus tres últimos viajes al Nuevo Mundo y en el último decidió no regresar con él. Junto a algunos de sus hombres, concluyó quedarse en aquellos parajes aún considerados como desconocidos. Moro apuntó a que la curiosidad y las ganas de experimentar cosas nuevas fueron los ejes del espíritu de este navegante. La aventura en un principio fue

4 Para Gruzinski la Modernidad se forjó en estos años y fue la mezcla de espacios y configuraciones políticas diversas que se conectaron mundialmente: "non plus par la construction de l'État-Nation ni par la marche vers l'absolutisme, pas plus qu'elle ne s'explique par le triomphe de la science et du rationalisme cartésien. Elle met en jeu d'autres espaces, d'autres configurations politiques -Monarchie catholique-, d'autres imaginaires et surtout d'autres acteurs qui ne son plus seulement des Européens, mais des Indiens comme Chimalphin, des Philippins, des Japonais, des mulâtres

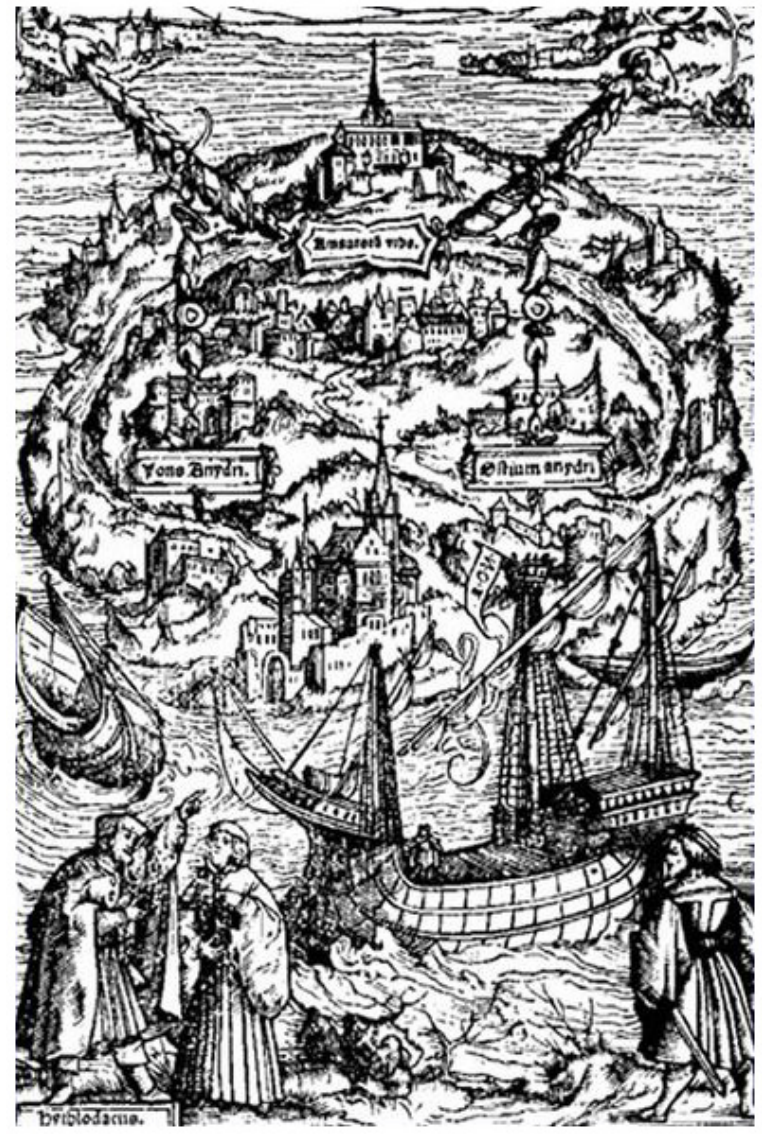

Fig. 1. Dibujo de Ambrosius Holbein para la edición de 1518 de La Utopía.

compleja para él y sus cuatro acompañantes. Debieron adaptarse a los modos de vida de los distintos grupos "indígenas" con los que tuvieron que convivir. En su narración, Hythloday señala que con el tiempo se ganaron la confianza de los diferentes pueblos que visitaron, lo que les permitió ser reconocidos como amigos y continuar una travesía caracterizada por no tener un destino fijo. Es así como estos hombres habría deambulado por múltiples "Estados y

d'Afrique ", (Gruzinski, 2004, p. 76).

No nos parece extraño que Moro haya situado a este marinero a la altura de un filósofo. Esto certifica la idea planteada por Sloterdijk que señala que la Modernidad floreciente para aquella época recaía en los geógrafos y los marinos. Para este pensador, fueron ellos y no los metafísicos los que dieron las directrices de las nuevas reflexiones sobre el tiempo y el espacio durante el siglo XVI. Ellos eran los verdaderos filósofos en aquellos tiempos (Peter Sloterdijk, 2004, p. 702). 
pueblos", sorprendiéndose de lo avanzado de sus instituciones y organizaciones socio-espaciales.

A pesar de que la aventura fue preciosa en cada lugar, hubo un antes y después para Raphael: haber visto y vivido en la isla de Utopía. Para él, no era una isla cualquiera, sus pobladores habían logrado crear un sistema social cuyo equilibrio y funcionamiento podía entenderse como "perfecto". Un sistema en donde todo aquello que podía crear alguna confusión entre los pares -como el tema de la propiedad de las tierras- se solucionó generando políticas de igualdad para todos.

\section{Dibujo de Ambrosius Holbein para la edición de 1518 de La Utopía.}

Hythloday exterioriza todo este desarrollo de una manera bastante estética (Marin, 2009, p. 10), sin embargo, esta armonía que entrelazaba la esfera política y social con lo espacial, también tuvo una relación con el caos. En efecto, la isla de Utopía habría encontrado el sendero hacia este supuesto "adelanto" a partir de una catástrofe. La catástrofe habría sido el momento desde donde se generó el sentido de este desarrollo idílico. La sociedad utopiana habría sido el producto del naufragio de un barco que transportaba romanos y egipcianos, los cuales habrían sido arrastrados por el mar hacia las costas de esta isla. Los sobrevivientes habrían sido capturados por los indígenas del lugar y con el pasar del tiempo, ambos grupos, pudieron establecer buenas relaciones. Así se inició un mestizaje e hibridación cultural que los habría llevado a esta "perfección social". En otras palabras, la aplicación de lo más "bello" del conocimiento europeo -según el autorcon la sabiduría de los nativos adquirida en la vida en la naturaleza fue lo habría forjado este avance. Ahora bien, Moro resaltó la situación del naufragio siempre como una situación en la

6 Traducción nuestra: "Es posible que después de este naufragio, la misma suerte llevó a alguno de nosotros a Utopía. No obstante, el recuerdo de esto se borró. Quizás, el futuro también olvidará mi estadía en esta isla afortunada, estadía que fue muy preciosa para los habitantes porque se apropiaron, por estos medios, de las más bellas invenciones de Europa

7 También se podría pensar que el hecho de vivir en una isla cual se nutrieron más bien los indígenas de esa isla que los propios europeos:

Il est possible qu'après ce naufrage, le même sort ait amené quelques-uns des nôtres en Utopie; mais le souvenir en est complètement effacé. Peut-être aussi la postérité oubliera-t-elle mon séjour dans cette île fortunée, séjour qui fut infiniment précieux pour les habitants, puisqu'ils s'approprièrent, par ce moyen, les plus belles inventions de l'Europe (More, 1842, p. 117) ${ }^{6}$.

\section{Forma y ubicación de la isla de Utopía.}

Pese a su gran capacidad de intuición sobre imaginar el mundo como si fuera un "globo", Moro no podía darle una forma concreta que no fuera la de una isla al territorio donde ocurría su historia. Esto porque hasta ese entonces no se sabía dónde terminaba el mundo y la esfericidad de la Tierra no estaba completamente comprobada. - La expedición de Magallanes pasa en 1520, aunque ya se pensaba que la Tierra era redonda $y$, como señalan algunos el Estrecho un secreto bien guardado (Dickson, 2014).

Sólo a partir de los itinerarios otorgados por las islas se podía, al menos, imaginar lo desconocido (Benat Tachot, 2001; Padrón, 2002, 2010) ${ }^{7}$.

Tanto en su disposición espacial como en el camino para llegar a ella, Utopía estuvo envuelta por geografías opuestas. Desde boscosos caminos hasta vastas llanuras la habrian circundado. En este sentido, sus descripciones respondieron a una puesta en escena que sintetizó los espacios explorados e imaginados para aquella época en diversos planos, pero en torno a un mismo centro geográfico y discursivo. Eso sí, un centro volátil en el que nunca se dejó en claro su posición geográfica ${ }^{8}$. Al respecto, fue el mismo Moro el que quiso dejar el misterio

motivara esta decisión.

8 Para Louis Marin, esto era parte de las impresiones que había en esos tiempos sobre cómo reflexionar sobre el Nuevo Mundo. Para él, la isla de Utopía reproducía parte de esto: «L'Utopie de More n'est ni l'Angleterre ni l'Amérique, ni l'Ancien ni le Nouveau Monde, mais l'entre-deux de la contradiction historique au début du XVIe siècle de l'Ancien et du Nouveau Monde ». Marin, op.cit, p.9 
instalado, ya que ninguno de sus personajes se interesó por develar el lugar exacto de su ubicación:

Vous pourrez aisément me tirer d'embarras en interrogeant Raphaël lui-même ou en lui écrivant. Et vous allez être obligé de le faire à cause d'un autre doute qui nous vient. Estce par ma faute, par la vôtre, par celle de Raphaël lui-même? Je ne saurais le dire. Nous avons en effet négligé de lui demander, et il n'a pas pensé à nous dire, dans quelle partie du nouveau monde Utopie est située. Je donnerais beaucoup pour racheter cet oubli, car j'ai quelque honte à ignorer dans quelle mer se trouve l'île au sujet de laquelle j'ai tant à dire 9 .

Marin llama a esta proposición de Moro un "simulacro de la síntesis", es decir, para él La Utopía fue un discurso que presentó una solución imaginaria, ficticia, a las contradicciones geográficas de aquella isla $\mathrm{y}$, por qué no, a las del propio período (Marin, 2009, p. 9, 257). Se podría decir que La Utopía se exhibió como un espacio de "fin de mundo" perfecto, que sirvió como un límite de un más allá, como una imagen de fondo -decorado- que permitía tranquilizar las expectativas que se tenían sobre lo inexplorado ${ }^{10}$. Esto porque situaba lo desconocido en un punto preciso, otorgándole características que representaban parte de los

9 Traducción nuestra: "Usted podrá fácilmente "sacarme de apuros" interrogando al mismo Raphael o escribiéndole. Y usted va a estar obligado a hacerlo a causa de otra duda que tenemos. ¿Fue mi culpa, la suya, o la de Raphael? No sabría decirlo. Nos descuidamos de pedirle, y no pensó en decirnos, en cual parte del nuevo mundo la Utopía está situada. Daría mucho por redimir este olvido, porque tengo vergüenza de ignorar en qué mar se encuentra la isla respecto de la cual tengo tanto que decir". Esta fuente se encuentra en La Utopía, pero no está adjunta al texto impreso, ver "Préface: du Traité de la meilleure forme de gouvernement", http://fr.wikisource.org/wiki/L\%E2\%80\%99Utopie/ Pr\%C3\%A9face (revisado 15 de abril de 2014).

10 En efecto, la Utopía como proposición literaria ayudó en la contención de una serie de sensibilidades que se comenzaron a desarrollar luego del descubrimiento de América y que sirvieron para apaciguar, en parte, los "conexiones planetarias" que se estaban desarrollando. No obstante, luego del descubrimiento del Estrecho conocimientos adquiridos hasta esos momentos.

En relación a la ubicación de esta isla, la descripción hecha por Raphael Hythloday posee detalles que dejan entrever dónde se habría encontrado esta tierra. La primera pista nos dice que esta isla se encuentra en el Nuevo Mundo. La confirmación de que la isla se encontraba bajo el Ecuador y que en gran parte del relato el autor se haya referido a un Nuevo Mundo nos permite afirmar que se trataba de los territorios o mares americanos.

Sous l'équateur, ajoutait Hythloday, et de part et d'autre, dans l'espace compris par l'orbite du soleil, ils ne virent que des vastes solitudes éternellement dévorées par un ciel de feu. Là, tout les frappait d'horreur et d'épouvante. La terre en friche n'avait d'autres habitants que les bêtes les plus féroces, les reptiles les plus affreux ou des hommes plus sauvages que ces animaux. En s'éloignant de l'équateur, la nature s'adoucit peu à peu; la chaleur est moins brûlante, la terre se pare d'une riante verdure, les animaux sont moins farouches. Plus loin encore, l'on découvre des peuples, des villes, des bourgs, où un commerce actif se fait par terre et par mer, non seulement dans l'intérieur et avec les frontières, mais entre des nations à grande distance (More, 1842 , p. 44) ${ }^{11}$.

Para mantener el misterio y por la inspiración

de Magallanes este espacio de contención virtual, es decir, aquel espacio en donde se acumulaban una serie de inquietudes, memorias y observaciones, se subyugó al poder de la bidimensionalidad de los mapas. Con respecto a la idea de "conexión planetaria" (Chaunu, 1969, p. 332)

11 Traducción nuestra: "Bajo el ecuador, agrega Hythloday, y de una parte a otra, en el espacio comprendido por la órbita del sol, ellos no vieron que de vastas soledades, devoradas por la eternidad de un cielo de fuego. Allí, todo les golpeaba con horror y terror. La tierra baldía no tenía otros habitantes que animales muy feroces, los reptiles los más horribles, u hombres más salvajes que estos animales. Alejándose del ecuador, poco a poco, la naturaleza se suavizaba; el calor quemaba menos, la tierra se adquiere un risuelo verdor, los animales son menos salvajes. Más lejos todavía, descubrimos personas, ciudades, pueblos y un comercio activo por tierra y mar, no solamente en el interior y con las fronteras, sino también entre naciones a gran distancia". 
que Moro tuvo del trabajo de Vespucio, como lo veremos prontamente, deducimos que la morfología de la isla de Utopía tuvo que ser exhibida como una tierra extremamente compleja tanto para entrar como para salir de ella. Una zona que no se podía ni se sabía traspasar, como hasta ese entonces sucedía lo mismo con la Tierra (Besse, 2003). De hecho, en el texto se señala que las geografías de la isla no permitían una forma de entrar que no fuera la de ir acompañado por un utopiano. La isla era presentada con límites y formas claras, pero fragmentada a la hora de querer penetrar en ella. En palabras de Louis Marin, "en el fondo, la Utopía no admite nada del exterior a ella misma: ella es a ella misma su propia realidad" (Marin, 2009, p. 137).

Ahora bien, esta realidad propia de la isla de Utopía fue artificial. En un principio este territorio estuvo adherido a un continente. Luego de un enfrentamiento entre los habitantes de aquellas zonas, se produjo este cambio. Moro dice que se trató de un enfrentamiento ganado por el Rey Utopus y su gente. Este Rey junto a sus soldados y los indígenas colonizados luego de la batalla, cortaron el istmo de quince millas que conectaba dicho terreno al continente $\mathrm{o}$ a las tierras antiguamente llamadas de Abraxa $^{12}$. Así se logró la independencia absoluta y la forma deseada de isla (More, 1842, p. 125).

$\mathrm{Si}$ consideramos las palabras utilizadas por Moro para describir tanto la isla en su interior como en sus alrededores, esto nos lleva a imaginar que las acciones de su historia sucedieron en el extremo sur del continente americano. El tema de los cambios radicales de la naturaleza en el trayecto hacia el sur, descritos por Hythloday -desde las temperaturas y paisajes salvajes a lugares "más dulces"-, y la inclusión de accidentes geográficos como istmos, golfos, estrechos y archipiélagos, nos permiten completar esta idea.

12 Moro mide en Pasuum las longitudes de la isla de Utopía. Esto equivale aproximadamente a dos pasos romanos, es decir, 1,48 metros. Agradecemos las precisiones a José Miguel de Toro quien realizó la verificación en la versión original en latín para nuestro trabajo.

13 Traducción nuestra: "La isla de Utopía tiene doscientas millas en su ancho más grande, situada en la parte media. Esta anchura se estrecha gradualmente y simétricamente del centro a ambas extremidades, de modo que la isla entera se redondea en un semicírculo de quinientas millas, y tiene la forma de una media luna, cuyos cuernos son alejados de aproximadamente once millas. El mar llena esta inmensa cuenca; tierras adyacentes que desarrollan en anfiteatro quebrantan allí el furor de los
Otro dato que nos permite imaginar dónde se habría ubicado esta isla, se desprende de la forma que Moro dio a esta. La isla de Utopía fue descrita como una gran medialuna, internamente forjada de estrechos y accidentes geográficos que permiten, entre otras cosas, parar el gran viento que hay en la zona.

L'île d'Utopie a deux cent mille pas dans sa plus grande largeur, située à la partie moyenne. Cette largeur se rétrécit graduellement et symétriquement du centre aux deux extrémités, en sorte que l'île entière s'arrondit en un demi-cercle de cinq cents miles de tour, et présente la forme d'un croissant, dont les cornes sont éloignées de onze mille pas environ. La mer comble cet immense bassin; les terres adjacentes qui se développent en amphithéâtre y brisent la fureur des vents, y maintiennent le flot calme et paisible et donnent à cette grande masse d'eau l'apparence d'un lac tranquille. Cette partie concave de l'île est comme un seul et vaste port accessible aux navires sur tous les points. L'entrée du golfe est dangereuse, à cause des bancs de sable d'un côté, et des écueils de l'autre. Au milieu s'élève un rocher visible de très loin, et qui pour cela n'offre aucun danger. Les Utopiens y ont bâti un fort, défendu par une bonne garnison. D'autres rochers, cachés sous l'eau, tendent des pièges inévitables aux navigateurs. Les habitants seuls connaissent les passages navigables, et c'est avec raison qu'on ne peut pénétrer dans ce détroit, sans avoir un pilote utopien à son bord (More, 1842, p. 124) ${ }^{13}$.

De una manera sideral esto podría coincidir con la disposición geomorfológica de una "isla" que posee una forma similar, la Isla Grande de Tierra del Fuego ${ }^{14}$.

vientos, mantienen alli el flujo tranquilo y apacible y dan a esta gran masa de agua la apariencia de un lago tranquilo. La parte cóncava de la isla es como un único gran puerto accesible a los buques en todos los puntos. La entrada del Golfo es peligroso debido a los bancos de arena en un lado y las rocas en el otro. En el centro se levanta un roquerio visible desde lejos, que no ofrece ningún peligro. Los utopianos han construido una fortaleza defendida por una buena guarnición. Otras rocas, escondidas bajo el agua, tienden trampas inevitables a los navegadores. Sólo las personas que habitan la Utopía conocen los pasajes navegables, y es por una buena razón que no podemos entrar en este Estrecho sin piloto que no sea utopiano". 
Ahora bien, sabemos que las formas disonantes que se dan en aquellas latitudes nos enseñan que podría tratarse de cualquier lugar. Sin embargo, fueron las grandes distancias propuestas por el inglés las que nos permiten superponer el nombre de la Isla Grande por sobre otros lugares.

Es cierto, no se trataría exactamente de las misma isla si consideramos las longitudes entre una y otra. El largo de la Isla Grande entre sus "cuernos" es aproximadamente 500 kilómetros, en tanto el de La Utopía es de solo un poco más de 16 kilómetros, o 11 mil pasuum como dice la versión original. En el ancho ocurre una diferencia también relevante entre ambas partes, ya que la Utopía sería mayor en talla en este sentido ${ }^{15}$.

A pesar de lo anterior, La Utopía deja entrever una similitud más bien en la forma y disposición geográfica en los alrededores de esta isla. La forma de medialuna, las dificultades de navegación y la extensión de sus tierras y el clima, nos permiten deducir que se podría tratar del mismo lugar, sin ser completamente equivalentes. Existiría una incongruencia matemática y de significados entre ambos, pero una coherencia simbólica entre sus formas. A su vez, esta coherencia se habría apoyado, entre otras cosas, en la noción de "fin de mundo" supeditada a ambos lugares, que habían dejado instalada expediciones como la de Américo Vespucio.

Foucault demostró que durante el siglo XVI, Occidente entendió su mundo a partir de la noción de lo parecido, delosemejante, esdecir, "elmundoseenvolvía en sí mismo". Las "cosas" para representarse y adquirir inteligibilidad debian compararse, contraponerse y analizarse con aquellas que eran similares (Foucault, 1966 , p. 32, 82 $)^{16}$. Ahora bien, para el francés la importancia de la semejanza de aquella época tiene que ser entendida en virtud de la imaginación, puesto que sin ella no podía producirse (Foucault, 1966, p.

14 La ubicación de la Isla Grande es la siguiente: $52,5^{\circ}$ Sur 52 27'17"S 69²5'23"O - (Punta Anegada, Chile) y su extremo sur es el cabo San Pío (Argentina), al sur del paralelo de $55^{\circ} \mathrm{S}$. Estas latitudes son similares a las descritas por Vespucio.

15 El ancho máximo de Utopía serían cerca de 296 kilómetros, en tanto el máximo de la Isla Grande de $150 \mathrm{~km}$.

16 Foucault reconoce cuatro tipo de formas de similitud que se dieron en el siglo XVI: Convenientia, Aemulatio, Analogie y las Sympathies. Si tuviésemos que realizar una comparación de estas cuatro formas con el texto de Moro, la isla de Utopía podría verse reflejada claramente en todas ellas. Ver Foucault, op.cit, pp. 32-40.
83). Si consideramos estas palabras, se podría deducir que tanto las particularidades, excentricidades y formas geográficas presentadas en la obra de Moro fueron informaciones procesadas por la imaginación de Moro que, probablemente, derivaron de un percepción exterior a él. Moro "asemejó" algo que le contaron o que leyó, y con ello creó una nueva representación espacial y/o propuesta política de cómo habitar el mundo o un "nuevo mundo". No podía hacerlo de otra forma. Para crear "nuevo mundo", tuvo que tomarse del Nuevo Mundo de aquel entonces. Tuvo la brillantez de reducir todas las observaciones, rumores y anotaciones en un espacio determinado, el cual según nuestras indagaciones fue un espacio semejante $y$ correspondiente a la zona magallánica, en especial, a la Isla de Tierra del Fuego. ${ }^{17}$

En referencia a la fuente de inspiración y de origen de sus ideas, fue el propio pensador el que precisó su procedencia: las anotaciones de un viajero tan connotado como Américo Vespucio. Eso sí, no se trató de cualquier tipo de notas, sino de aquellas que este dejó sobre sus viajes a América.

\section{La influencia de Américo Vespucio}

Moro tuvo acceso a los escritos de Vespucio que desde 1507 circulaban por Europa y que habían sido representados en obras como las de Kunstmann II, Maiollo (1504), Caverio y Waldseemüller (1507) (Vespucci, 2005).

Se ha discutido bastante hasta qué punto de América -latitud sur- habrían llegado Vespucio y los portugueses durante los cuatro viajes que habrian realizado entre 1497 y 1504. También se ha discutido si realmente fue este navegante el que escribió las anotaciones que lo hicieron merecedor de que un continente llevara su nombre ${ }^{18}$. Como nuestra reflexión está dentro del plano de la representación,

17 Para tener una visión panorámica sobre la historia y geografía de la zona, ver los trabajos de Mateo Martinic (1992, 2002).

18 Cf. Rolando Laguarda por medio de un riguroso estudio ha puesto en tela de juicio si el contenido de las cuatro cartas más conocidas de Vespucio fueron escritas por él, lo que pone en entredicho además las latitudes a las que posiblemente navegó. Para ello, analizó la grafía y comparó los documentos con otras cartas y representaciones del período. El autor deja entrever que solo las cartas del 4 de junio de 1501 y la de 1502 escrita desde Lisboa fueron únicamente las verdaderas. Ver en espacial, los capítulos IV y V (Laguarda, 1982). Laguarda también toma como referencia el texto de Magnaghi (1924). 


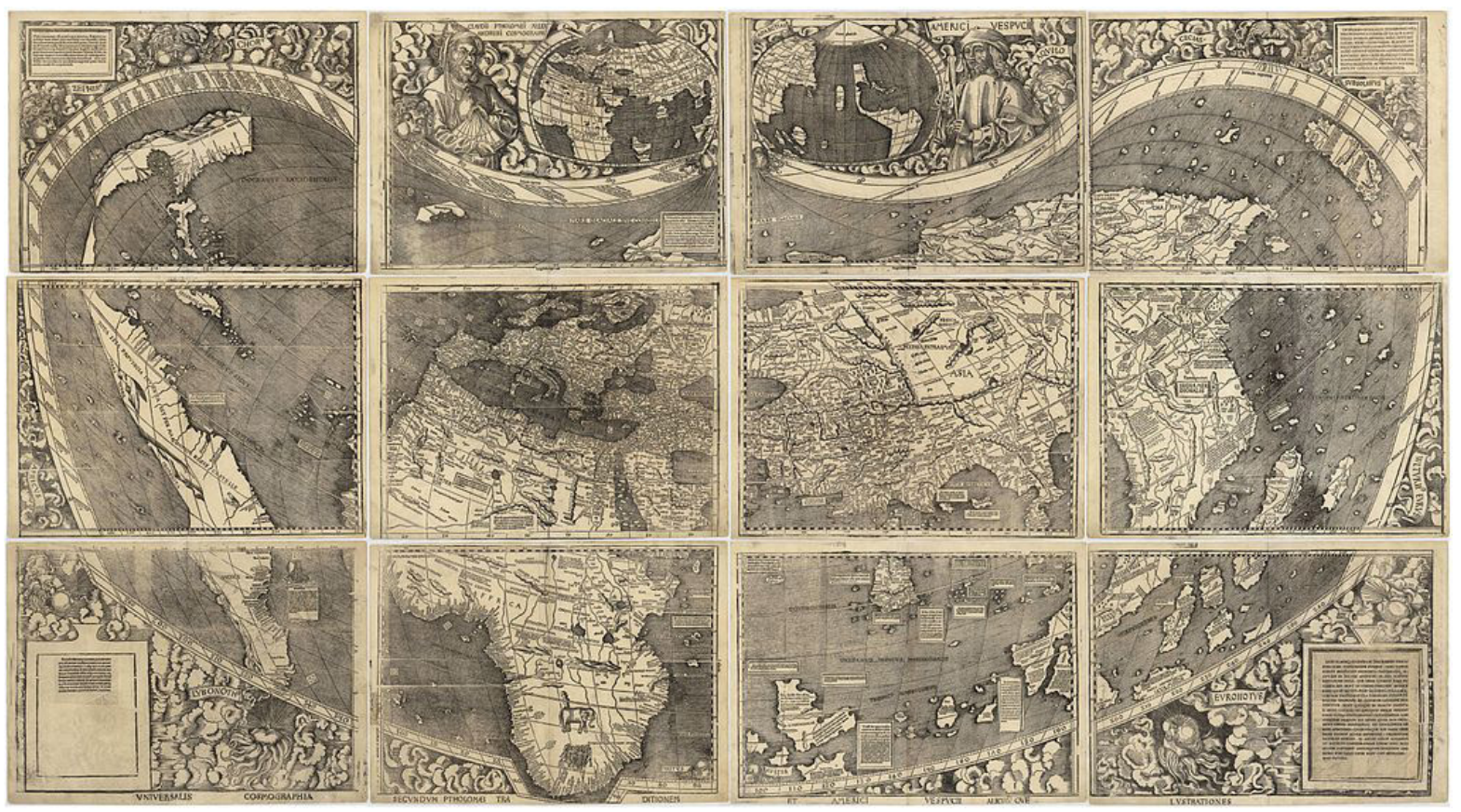

Fig. 2. Universalis Cosmographia, $1507^{20}$

nos interesa abordar el discurso "sobreviviente", es decir, que fue Vespucio el protagonista y delineador de estas temáticas presentadas como incógnitas ${ }^{19}$. A su vez, más allá de las dudas que se puedan generar al respecto, nuestra propuesta metodológica se ampara en lo que cita nuestra fuente de origen -libro de La Utopía-. En él queda marcado, sin ninguna duda, el nombre de Vespucio como el propulsor de estos descubrimientos y de su llegada hasta el extremo sur del continente americano.

18 Cf. Rolando Laguarda por medio de un riguroso estudio ha puesto en tela de juicio si el contenido de las cuatro cartas más conocidas de Vespucio fueron escritas por él, lo que pone en entredicho además las latitudes a las que posiblemente navegó. Para ello, analizó la grafía y comparó los documentos con otras cartas y representaciones del período. El autor deja entrever que solo las cartas del 4 de junio de 1501 y la de 1502 escrita desde Lisboa fueron únicamente las verdaderas. Ver en espacial, los capítulos IV y V (Laguarda, 1982). Laguarda también toma como referencia el texto de Magnaghi (1924).

19 Un texto que habla sobre este tópico y las polémicas en torno a la figura de este cosmógrafo es el de Stefan Zweig (Zweig, 2010).

20 Martin Waldseemüller, UniversalisCosmographia, Estrasburgo, 1507. En este planisferio, ubicado en la Library of Congress en Washington, se nombra por primera América -tomando las anotaciones de Vespucio
A pesar de las confusiones en torno a la figura del florentino, los relatos que habría realizado sobre el continente americano fueron claros y consideraron a la zona magallánica. La utilización de elementos y saberes aprendidos los llevaron a calcular de manera casi exacta a qué latitud se encontraron en cada lugar. El propio cosmógrafo narró que los vientos los llevaron hacia los $52^{\circ}$ grados de latitud sur durante su tercer viaje $e^{21}$.

En una de sus cartas más famosas conocida

-a las tierras descubiertas por Colón. Cf. John Hessler (2005, 2012). Este último autor realizó un estudio de carácter matemático que permitió establecer la similitud en la propuesta de Waldseemüller con la geografía de América, a pesar de no tener conocimientos exactos sobre aquellas zonas para aquel entonces. En sus textos comenta que el dibujante logró proyectar correctamente ciertos trazos aún imaginados como la parte oeste de Chile. A su vez, deja entrever que esta representación del mundo no se realizó en estas fechas, sino un poco después. Por otra parte, para el caso chileno, Alejandra Vega se pregunta sobre cómo fue capaz de proponer geografías que no se conocían para aquel entonces (Vega, 2014, p. 84-86).

21 Un contemporáneo de Vespucio, el alemán Valentin Ferdinad's señaló que Vespucio habría alcanzado incluso los 53 (Formisano, 1985) (Vespucci, 2005, p. 30, 221) (Laguarda Trías, 1982). 
como Lettera, el navegante certifica el hecho de que llegó al grado $52^{\circ}$. Dentro del escrito se puede apreciar que coinciden las geografías y clima de aquellas zonas:

"Après avoir fait nos provisions sur cette terre, nous commençâmes à naviguer par vent du sudest, et ce fut le 15 février, alors que le soleil se rapprochait de l'équinoxe et de notre hémisphère du Septentrion. Nous naviguâmes tant sous ce vent que nous atteignimes une latitude telle que le pôle Sud était éloigné de notre horizon de $52^{\circ}$. En effet, nous ne voyions plus les étoiles de la petite Ourse ni celle de la Grande et nous nous étions déjà éloignés de 500 lieus environ du port d'où nous étions partis par ce vent de siroco. Nous étions le 3 avril. Ce jour-là une tempête se leva en mer, si forte qu'elle nous obligea à amener totalement nos voiles et que nous continuâmes avec les mâts sans voiles par grand vent sud-ouest. Les vagues étaient énormes et la tourmente grondait au point que la flotte tout entière éprouvait une grande frayeur. Les nuits étaient très longues et celle que nous eûmes le 7 avril, dura 15 heures, car le soleil se trouvait à la fin de la constellation du Bélier et parce que dans cette région on était en hiver Votre Magnificence peut le calculer. Alors que nous étions dans cette tempêté, le 7 auril, nous aperçûmes une terre nouvelle que nous longeâmes pendant environ 20 lieues. Nous trouvâmes que c'était une côte sauvage et nous n'y vîmes aucun port et aucun habitant et je crois que c'était parce qu'il y faisait un tel froid que personne dans la flotte ne pouvait s'en protéger ni le supporter» ${ }^{22}$.

Cabe recordar que la inflexión en la obra de Moro con respecto a su filosofo-marino se dio entre el tercer y cuarto viaje. Se subentiende que

22 Lettre D'Americ Vespuce sur les îles nouvellement découvert dans ses quatre voyages. Lisbonne, 4 septembre de 1504 ", Vespucci, op.cit., pp. 199-200. Traducción nuestra: "Después de provisionarnos en esta tierra, comenzamos a navegar con viento sureste, y fue el 15 de febrero, cuando el sol se acercaba al equinoccio y a nuestro hemisferio Norte. Navegamos bajo este viento que alcanzamos una latitud tal que el Polo Sur aún estaba muy lejos de nuestro horizonte de $52^{\circ}$. De hecho, no veíamos la Osa Menor o ni la Grande y ya estábamos cerca de 500 leguas lejos del puerto del cual habíamos partido bajo un el viento siroco. Era el 3 de abril. Ese día una tormenta en elevó el mar, tan fuerte
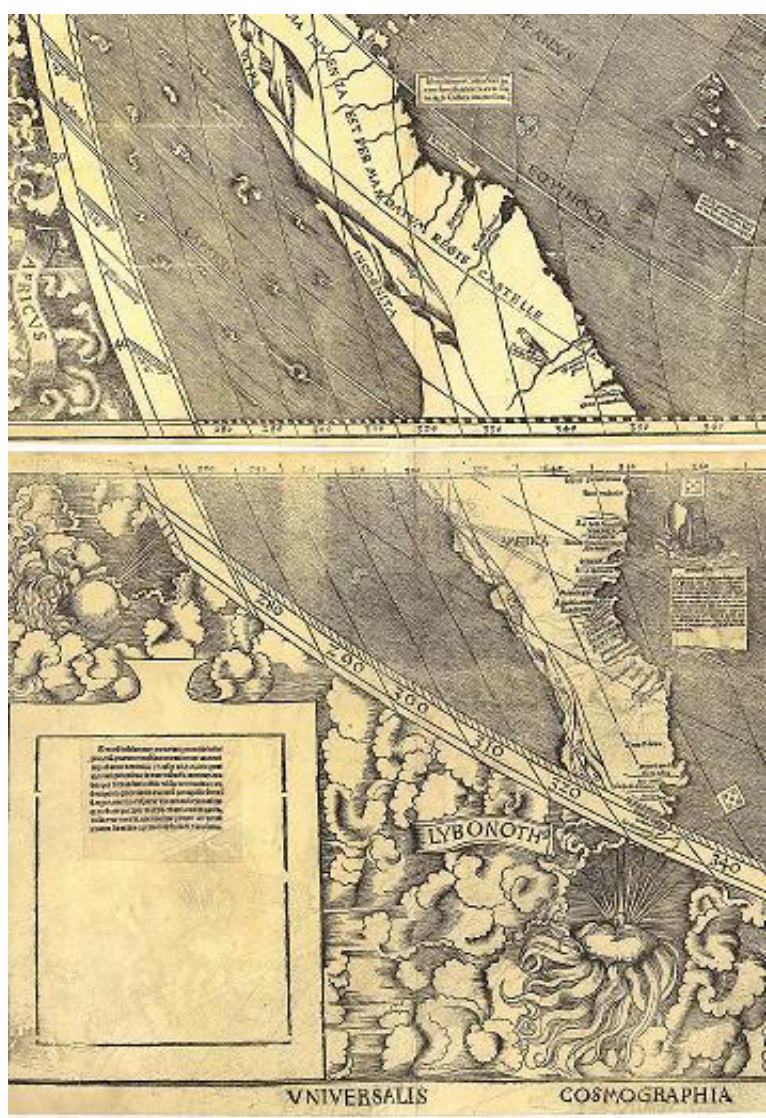

Fig. 3. Esta imagen que ampliamos de Universalis Cosmographia muestra la concepción del continente americano para esos años y la importancia portuguesa. Al final de América hay una bandera que corresponde a la del reino portugués. Las tierras del extremo sur del continente se dan como existentes, pero se omiten dentro de la imagen por medio de un clima

adverso (viento y nubes) que no permite ver el más allá.

luego de maravillarse en el tercer viaje de Vespucio, Hythloday decidió quedarse en aquellas tierras desconocidas en las terminaron descubriendo junto a sus compañeros la isla de Utopía.

que nos obligó a recoger nuestras velas y continuamos con los mástiles sin velas bajo un gran viento del suroeste. Las olas eran enormes y la tormenta rugió al punto que toda la flota tuvo un gran temor. Las noches eran muy largas y la vivimos el 7 de abril, duró 15 horas, porque el sol estaba en el extremo de la constelación de Aries y porque en esta zona era invierno, Su Majestad lo puede calcular. Mientras estábamos en esta tormenta, el 7 de abril, vimos una nueva tierra que bordeamos durante unas 20 leguas. Nos pareció que era una costa salvaje. No vimos ningún puerto y ningún habitante y creo que fue porque hacía un frio tal ninguna persona de la flota podía ni protegerse ni soportarla". 
Además de las geografías descritas por el pensador inglés, existen ciertos detalles que permitirían reforzar que la isla de Utopía fuera un receptáculo de notas de viajes reales ocurridos en la época. El hecho de que el protagonista de la obra tuviera nacionalidad portuguesa no fue algo menor. Es más, Gilles destacó la nacionalidad de Raphael Hythloday como algo importante para entender el tenor de los hechos que se estaban exponiendo. Se reseñó al viajero como un hombre que abandonó muy joven su casa porque lo devoraba la pasión por recorrer el mundo. Dentro de esta pasión se habría aferrado a Vespucio y participado en tres de sus cuatro viajes (More, 1842, p. 41-42) ${ }^{23}$.

Esto permite confirmar, nuevamente, que Moro leyó atentamente a Vespucio y que estudió muy bien los viajes realizados por los portugueses, que para esos años eran la potencia en este tipo de empresas y que marcaban los pasos de la geopolítica mundial (Subrahmanyam, 2013; Sallamann, 2003).

\section{Consecuencias de esta posible equivalencia}

Ce n'est pas le rapport entre l'utopie, comme prévision, et l'avenir à prévoir qui est au centre des préoccupations de l'historien. Il se demande plutôt comment, de quelle manière spécifique, la réalité sociales d'un certain présent se traduit et se manifeste dans les utopies et par les utopies, comment les utopies participent au présent en s'efforçant de le dépasser ${ }^{24}$.

El objetivo principal planteado con este breve escrito era mostrar las aproximaciones entre las formas geográficas utilizadas por Moro

23 Otro detalle expuesto fue vuelta de Raphael y sus compañeros a Europa luego de pasar por Utopía. Gracias a una serie de encuentros fortuitos con navegantes portugueses estos habrían vuelto al viejo continente. Dentro del relato se nombran ciertos lugares que otorgan veracidad a la narración de Moro. Ejemplo de esto son Taprobane y Calicut. Estos parajes coinciden con lo sucedido con las expediciones Vasco de Gama y Pedro Alvares Cabral para esos mismos años. De hecho, en el diario de Pedro Alvares Cabral, escrito por un piloto anónimo (relación de Cretico), se lee que al regreso de la expedición estos marineros pasan por los mismo lugares citados por Moro: "Llegamos al cabo de Buena Esperanza, día de Pascua de Flores, y para describir su isla de Utopía -inspiradas en las anotaciones de Vespucio-, con la zona que comprende la Isla Grande de Tierra del Fuego $y$ sus alrededores. Sabemos que existen algunos vacíos en las informaciones que no nos permiten señalar de manera categórica que esto pudo ser así. Sin embargo, nos parece fundamental dejar constancia - y con esto develamos un objetivo menos visible de nuestro estudio-, que para esos años y hasta finales del siglo XVI, describir y cartografiar la zona patagónica fue un ejercicio que vinculó sensibilidades relacionadas con lo incognito y con geografías imaginadas que se crearon antes de que los europeos intentasen posicionarse de manera "real" en la zona, es decir, luego del descubrimiento del estrecho de Magallanes en 1520.

Probablemente, poco importa si la forma de la isla de Utopía se inspiró totalmente en la Isla Grande de Tierra del Fuego. Lo relevante es que la geomorfología descrita en la primera se asemejó con las formas geográficas de de la segunda y de toda la zona alrededor de ella. Creemos que esto no fue menor, puesto que permitió que posteriormente se traspasaran algunas de las sensibilidades en torno a los contenidos y espacios de esa creación literaria al territorio real. En otras palabras, las percepciones que se tuvieron sobre las tierras comprendidas entre el estrecho de Magallanes y la Isla Grande -luego de ser descubiertas- fueron correspondientes a parte de los significados y pasajes expresados por Moro en su obra. Por tanto, creemos que la supuesta equivalencia no sólo pudo darse en referencia a las formas geográficas y la "sensación ambiental" de la zona, sino también en la manera cómo se conjugaron las percepciones de ambas de forma

alli hallamos buen tiempo, con el que pasamos adelante, y abordamos en la primera tierra junto a Cabo Verde, que se llama Besenegué, en donde hallamos tres navíos, que el rey de Portugal había mandado para descubrir la tierra nueva que nosotros habíamos hallado cuando íbamos para Calicut", Laguarda Trías, op. cit, p. 16.

24 Nuestra traducción: "No es la relación entre la Utopía como previsión y el avenir a prever lo que está en el centro de preocupación de un historiador. Él se pregunta más bien cómo, de qué manera específica, la realidad social de un presente determinado se traduce y manifiesta en las utopías y por las utopías; o también le interesa saber cómo las utopías participan en el presente e intentan sobrepasarlo", (Baczko, 1971, p. 356-357). 
similar en el tiempo.

Dentro de estas percepciones podríamos distinguir algunas herencias directas que la zona magallánica absorbió y que se vieron reflejadas algunos años después. De manera global reconocemos tres grandes legados conectados entre sí que cruzaron todo el siglo XVI: el fantasma de la catástrofe bajo la idea de naufragio, la imagen de insularidad y las expectativas riquezas.

En La Utopía se reconoce el naufragio bajo una doble dimensión. La primera como el origen de todo, es decir, se presentó como parte de una experiencia necesaria para poder descubrir y construir un lugar maravilloso (caso del naufragio de romanos y egipcianos). Una segunda arista, fue que el naufragio en tanto desastre, fue una posibilidad a considerar dentro de la propia isla en caso de no tener conocimiento de cómo traspasarla. Moro en reiteradas ocasiones precisó que sólo los utopianos tenían la destreza para traspasar los múltiples accidentes geográficos.

Si esto lo traslapamos a lo que pasó en la zona desde 1520 hasta finales del siglo XVI, claramente vemos posibles nexos. Como se sabe, desde la primera travesía los parajes asociados al estrecho de Magallanes y a la Tierra del Fuego- cargaron con la imagen negativa de los naufragios. Esto porque los riesgos y desventuras que se corrieron durante el viaje de Magallanes, -frío, falta de agua dulce, comida, la pérdida de una de las naves y la deserción de otra-, promovieron que desde estos tiempos dichos territorios fuesen supeditados a una imagen de riesgo y catástrof $e^{25}$. Se superpuso la imagen de dificultad y pérdida antes que la del éxito de haber descubierto un pasaje que conectaba al globo.

Carlos V y sus colaboradores no facilitaron las cosas para que se realizaran expediciones,

25 Para mayores detalles, Mariano Cuesta Domingo, "Alonso de Santa Cruz, cartógrafo y fabricante de instrumentos náuticos de la Casa de Contratación", Revista Complutense de Historia de América, v. 30 (2004), pp. 7-40.

26 Ejemplos de expediciones ordenadas por Carlos $\mathrm{V}$ y que fracasaron fueron las dirigidas por Frey García Jofré de Loaysa y Sebastian Elcano en el año 1526, y la de Sebastián Caboto en el mismo año.

27 Las secuelas de esto fue que redefinieron las formas de utilizar los medios económicos -incluso privados - en las nuevas conquistas americanas y que se establecieran

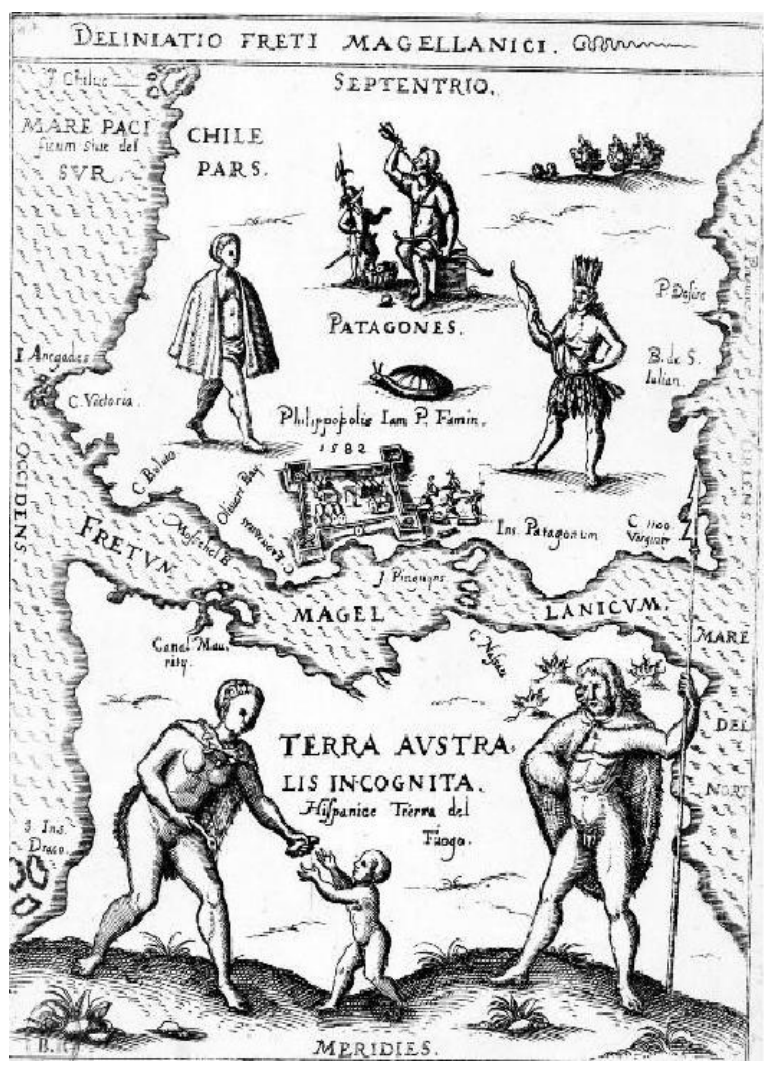

Fig. 4. Levin Hulst dit Levinus, Illustrations de Sechste teil Kurtze Warhafftige Relation Beschreibung der Wunderbasten vier Schiffarten, Nuremberg, Edición C. Lochner, 1602, p. 10.

luego de que algunos intentos tanto de carácter oficial como privados no tuvieran éxito y con ello se perdieran numerosos medios ${ }^{26}$. Esto no quiere decir que la búsqueda de un paso a la China y/o Indias se detuvo, sino que los españoles tomaron la decisión de buscar otros pasajes, eso sí, más al norte de América ${ }^{27}$. En efecto, hasta 1578 no se pudo atravesar el Estrecho de Magallanes por no saber cómo atravesarlo ni devolverse (sensación similar expuesta en el texto de Moro) ${ }^{28}$.

nuevas regulaciones políticas sobre los límites de las jurisdicciones entre los ibéricos, como lo fue el "Tratado de Zaragoza" en 1529. Eso sí, cabe precisar que para esos años la Monarquía no puso todos sus esfuerzos en penetrar aquel fin de mundo, ya que Carlos $\mathrm{V}$ estaba preocupado de una gran cantidad de conflictos comunidades de Castilla, Turcos, etc.- que lo distrajeron del potencial de las nuevas tierras descubiertas, como también tenía la presión de los conquistadores del norte de América que no les gustaba que hubiese otra posibilidad de pasar a las Indias que no fuera por Panamá. 


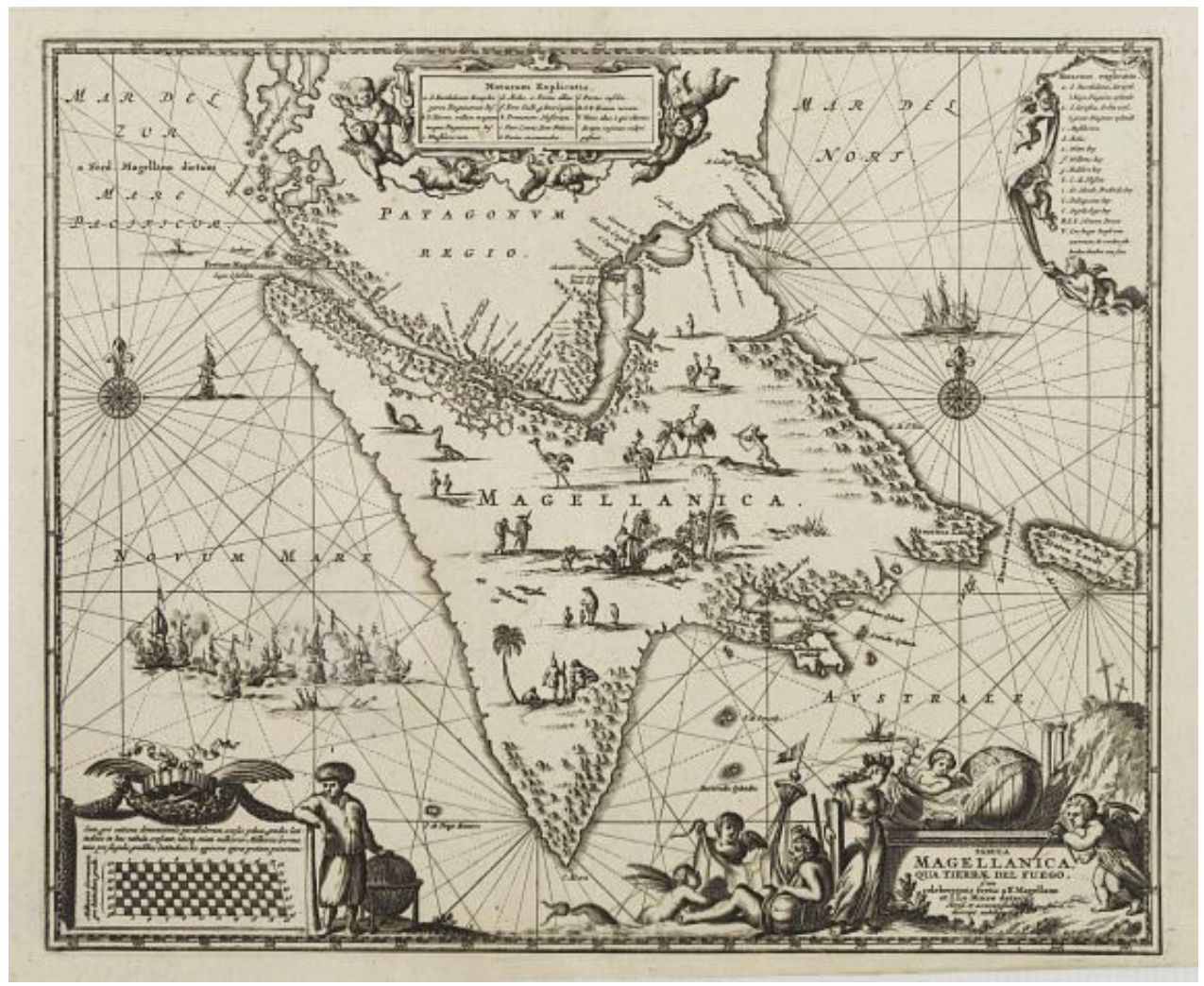

Fig. 5. Apud Joennem Janssonium, Tabula Magellanica qua Tierrae del Fuego, cum celeberrimis fretis a

F. Magellano et I. le Maire detectis novissima et accuratissima descriptio exhibetur, Amsterdam, 1671.

El escenario anterior, nos permite introducir la segunda herencia. Las decisiones políticas tomadas por la corona durante estos primeros tiempos consolidaron un escenario de exclusión sobre estos parajes de "fin de mundo". Este paso al costado por parte de la Monarquía de querer apropiarse de esta zona, suscitó un cambio de significación hacia esta geografía recientemente descubierta. No pesó ni el afán de ganancia ni la curiosidad. La zona fue aislada y presentada casi como una tierra incógnita, prohibida y asociada a lo negativo de la expedición de Magallanes y al fracaso de las que las siguieron.

Se podría decir que políticamente se "aisló" esta parte del Nuevo Mundo, otorgándole funcionalmente y discursivamente una propiedad de isla, es decir, un espacio de tierra delimitado independiente de otros, pese a ser parte de un continente y un pasaje entre dos océanos. La

28 Sobre los naufragios, sobrevivencias y los significados que adquiere la "Patagonia" durante el siglo XVI, ver monarquía española hizo algo similar a lo que hizo el rey Utopus, fragmentar los territorios y construir artificialmente una insularidad en la zona, pese a que ni el Estrecho ni la Isla Grande de Tierra del Fuego fueron un solo trozo de tierra, sino más bien un conjunto de caminos y accidentes geográficos. Especulamos que se superpusieron las percepciones negativas que se tenían -resumidas en la política de aislamiento- a la pluralidad de las geografías que conforman dichos territorios. Esto habría provocado que aquella multiplicidad de formas geográficas se convirtiera en una sola imagen, singular, a la cual era más fácil aislar. De hecho, las representaciones cartográficas y cosmográficas posteriores "cargan" con muchas de estas herencias.

Esta insularidad que distinguimos no es una mera interpretación de nuestra parte, sino estuvo legitimada por los propios cartógrafos y marinos de

(Jocelyn-Holt, 2004, p. 27-123). 
aquellos años que promovieron por muchos años esta mirada. Alonso de Santa Cruz quien vivió gran parte del siglo XVI (1505-1567) y trabajó directamente con la Junta de Pilotos de la Monarquía (Oïffer, 2012) ${ }^{29}$, dio a la zona asociada al Estrecho de Magallanes una definición de isla en su Islario general de todas las islas del mundo. De hecho, dentro de su trabajo coincide con ser la última isla del mundo: "Esta tierra, que por la parte austral hace el estrecho llamado Magallanes, aunque no del todo y precisamente descubierta, consta ser la isla mayor que hay en el mundo, la cual es la más próxima tierra al antártico... "(Santa Cruz, 1984, p. 366).

Una última herencia que se puede tener en cuenta fue el tema de las expectativas de riqueza y la idealización de los espacios. Una de similitudes entre Vespucio y Moro fue que ambos relatos motivaron una serie de expectativas de riquezas inauditas en estas zonas descritas como de "fin de mundo" o antípodas. Vespucio destacó en más de un escrito que traspasó la zona de los $50^{\circ}$, subrayando no sólo la difícil navegación, sino también las bondades de una tierra exuberante, de grandes montañas y valles extendidos. Una tierra marcada por sus riquezas en oro y de personas muy agradables según su relato, pero difíciles de describir. El metal precioso encontrado en aquellos parajes y que no fue traído a Europa por aquella tripulación -para evitar problemas, según Vespucio-, abrió una brecha para imaginar que grandes cantidades podrían ser explotadas si se lograba localizar un lugar seguro donde posicionarse en aquellos territorios:

Nous naviguâmes si longtemps en suivant

29 Para mayores detalles, Mariano Cuesta Domingo, "Alonso de Santa Cruz, cartógrafo y fabricante de instrumentos náuticos de la Casa de Contratación", Revista Complutense de Historia de América, v. 30 (2004), pp. 7-40.

30 Esta información se encuentra en su escrito Nouveau Monde. Rolando Laguarda señala la existencia de más de 42 ediciones de esta carta con nombres distintos, uno de ellos se llama "Ora Antartica". Laguarda, op.cit, p. 26. Traducción nuestra: "Navegamos mucho tiempo siguiendo esta orilla, después de haber pasado el trópico de Capricornio, encontramos el horizonte el más alto hacia el Polo sur a $50^{\circ}$, y nos acercamos al Círculo Polar Antártico a los $17^{\circ} 30$. Lo que vi y experimenté de la naturaleza de estas personas, de sus costumbres y su afabilidad, de la fertilidad de la tierra, del aire limpio, de la disposición del cielo y cuerpos celestes... voy a hablar primero de estas personas. En estos países, nos encontramos con muchas ce rivage, qu'après avoir dépassé le tropique du Capricorne, nous trouvâmes l'horizon le plus haut vers le pôle antarctique à $50^{\circ}$ et nous approchâmes du cercle antarctique à $17^{\circ} 30^{\prime}$. Ce que j'ai vu et connu de la nature de ces peuples, de leurs coutumes et de leur affabilité, de la fertilité de la terra, de la salubrité de l'air, de la disposition du ciel et corps célestes... Je parlerai donc d'abord de ces peuples. Dans ces pays, nous avons rencontré un si grand nombre de gens qu'il serait impossible à quiconque de les dénombrer, comme il est dit dans l'Apocalypse... La terre de ces pays est très fertile et agréable, avec de nombreuses collines, des montagnes, des vallées infinies et de très grands fleuves, des sources salubres avec des eaux abondantes, des forêts denses et impénétrables et pleines à foison de toutes sortes de bêtes sauvages...On n'y trouve aucune sorte de métaux, excepte l'or qui est abondant dans ces pays, bien que nous n'en ayons pas rapporté dans cette première navigation (Vespucci, 2005, p. 138) ${ }^{30}$.

Se puede encontrar una similitud a estas palabras en los escritos de Moro. De hecho, hablaba de las grandes riquezas de los utopianos y del desinterés que provocaba en ellos el oro, al encontrarse en cuantiosas cantidades en aquella isla (More, 1842, p. 168-172). Sin duda, ambos relatos abrieron una expectativa sobre aquellos parajes inexplorados, convirtiendo ese "fin de mundo" en un espacio añorado y deseable ${ }^{31}$, en una suerte de Eldorado o en una Ciudad de los Césares como se imaginó durante los años que

personas que sería imposible que alguien pudiera contarlas, como se dice en el Apocalipsis ... La tierra de este lugar es muy fértil y agradable, con muchas colinas, montañas, valles interminables y muy grandes ríos, fuentes de agua potable y abundante, de bosques densos e impenetrables, y llena de toda clase de fieras salvajes ... no se encuentra ningún tipo de metal, a excepción del oro que es abundante en estos lugares, aunque no trajimos con nosotros en este primer viaje".

31 Desde la primera expedición la zona adquirió un renombre a partir del oro esperado como de la manera como se denominaban los lugares que la componían. Elcano se refirió a algunas islas del sector como lugares llenos de oro (Castro, 2010, p. 614), en tanto, Magallanes, "entre lágrimas" como decía Pigafetta, denominó a uno de los cabos de donde recogieron pertrechos, como el cabo Deseado (Castro, 2010, p. 109). 
prosiguieron a esta zona ${ }^{32}$. Esto no fue menor, puesto que hasta fines del siglo XVIII de manera "oficial" se crearon expediciones para llegar hasta estas supuestas ciudades míticas que se encontrarían en esas latitudes del mundo (Urbina, 2009, p. 153-190).

\section{BIBLIOGRAFÍA}

Ainsa, F. (1992). Historia, utopía y ficción de la Ciudad de los Césares. Metamorfosis de un mito. Editorial, Alianza Madrid.

Baczko, B. (1971). Lumières et utopie. Problèmes de recherche. Annales, 26 (2), 356-357.

Benat Tachot, L. (2001). De l'ile à l'Islario: fonction et statut de l'île dans l'écriture de la conquête. En Trec, E (dir). Bout du voyage, l'ile: Mythe et réalité (56-87). Reims : Publications du Centre de Recherche VALS, Presses Universitaires de Reims.

Besse, J-M. (2003). Les grandeurs de la Terre. Aspects du savoir géographique à la Renaissance. Lyon : ENS Éditions.

Bradshaw, B. (1981). More on Utopia. The Historical Journal, 24 (1), 1-27.

Castro, X. (2010). Le voyage de Magellan (1519-1522). La relation d'Antonio Pigafetta \& autres témoignages. Paris : Chandeigne, coll. "Magellane».

Chaunu, P. (1969). L'Expansion européenne du XIIIe au XVe siècle. Paris : Nouvelle Clio.

Estellé, P., Couyoumdjian, R. (1968). La ciudad de los Césares: origen y evolución de una leyenda. Historia, $n^{\circ} 7,283-$ 309.

Dickson, P. (2014). The Magellan myth: reflections on Columbus, Vespucci, and the Waldseemueller map of 1507. Mount Vernon, Ohio: Printing Arts Press.

Elias, N. (2014). L'utopie. Paris: Éditions la Découverte.

Foucault, M. (1966). Les mots et les choses. Une archéologie des sciences humaines. Paris: Gallimard.

Goodey, B. (1970). Mapping "Utopia»: A Comment on the Geography of Sir Thomas More. Geographical Review, 60 (1), 15-30.

Gruzinski, S. (2004). Les quatres parties du monde. Histoire d'une mondialisation. Paris: Éditions de La Martinière.

Hessler, J. (2005). "Warping Waldseemüller: A Cartometric Study of the Coast of South America as Portrayed on the 1507 World Map". Coordinates, Series A, N 4 (http://purl.oclc.org/coordinates/b4.htm).

32 Referencias indiscutibles sobre estos tópicos que nos permiten contraponer nuestras ideas son los textos de
Hessler, J., Van Duzer, C. (2012). Seeing the World Anew. The radical visión of Martin Waldseemüller's 1507 \& 1516 worlds maps. Washington, Florida: Library of Congress, Levenger Press.

Jocelyn-Holt, A. (2004). Historia General de Chile. Tomo II. Santiago: Editorial Universitaria.

Laguarda Trías, R. (1982). El hallazgo del Río de la Plata por Amerigo Vespucci en 1502. Montevideo: Biblioteca de la Academia Nacional de Letras.

Magnaghi, A. (1924). Amerigo Vespucci. Roma: AGAR. (Tomo I).

Marin, L. (1993). Frontiers of Utopia: Past and Present. Critical Inquiry, 19 (3), 397-420.

Marin, L. (2009 [1973]). Utopiques : jeux d'espaces. Paris : Les Éditions de Minuit.

Martinic, M. (1999). Cartografía magallánica 1523-1945.

Punta Arenas: Ediciones de la Universidad de Magallanes.

Martinic, M. (1992). Historia de la región magallánica. Punta Arenas: Ediciones de la Universidad de Magallanes.

More, T. (1842 [1516]. L'Utopie. Paris : Paulin.

More, T. (1987). L'Utopie. Paris: Flammarion.

Moretti, G. (1993). "The Other World and the 'Antipodes': The Myth of the Unknown Countries between Antiquity and the Renaissance". En Haase, W., Reinhold, M. The Classical Tradition in the Americas. Berlin, New York: De Gruyter, 1993, Tomo I, 241-84.

Oïfer, A. (2012), "El Islario general de todas las islas del mundo (1560) de Alfonso de Santa Cruz o la ciencia cosmográfica en la España de Felipe II: en el Nuevo Mundo, el Caribe insular". Savoirs en Prisme, 1, 169187.

Padrón, R. (2002). Mapping Plus Ultra: Cartography, Space, and Hispanic Modernity. Representations, 79, 28-60.

Padrón, R. (2004). The Spacious Word: Cartography, Literature, and Empire in Early Modern Spain. Chicago: University of Chicago Press.

Padrón, R. (2010). Las Indias olvidadas: Filipinas y América en la cartografía imperial española., En Universidade de São Paulo $3^{\circ}$ Simpósio Iberoamericano de História da Cartografia Agendas para a História da Cartografia Iberoamericana. São Paulo.

Raitiere, M. (1973). More's Utopia and The City of God. Studies in the Renaissance, 20, 144-168.

Randles, W. (1961). "Le Nouveau Monde, l'Autre Monde et la Pluralité des Mondes". En Congresso Internacional de Historia dos Descobrimentos. Lisboa: Comissão

(Ainsa, 1992; Estellé y Couyoumdjian, 1968) 
Executiva das Comemorações do $\mathrm{V}$ Centenário da Morte do Infante D. Henrique, vol. IV, 347-385.

Rotterdam, E (1511). L'Éloge de la Folie. Bale: Johann Froben

Santa Cruz, A. (1984). Alonso de Santa Cruz y su obra cosmográfica.Madrid: Consejo Superior de investigaciones científicas, Tomo II.

Shephard, R. (1995). Utopia, Utopia's Neighbors, Utopia, and Europe. The Sixteenth Century Journal, 26 (4), 843856.

Sallamann, J-M. (2003). Géopolitique du XVIe siècle, 14901618. Paris : Points.

Sloterdijk, P. (2004). Esferas II. Globos. Ediciones Siruela, Madrid.

Subrahmanyam, S. (2013). L'empire portugais d'Asie, 1500-
1700. Paris: Points.

Urbina Carrasco, M. (2009). La frontera de arriba en chile colonial. Interacción hispano-indigena en el territorio entre Valdivia y Chiloé e imaginario de sus bordes geográficos, 1600-1800. Valparaíso: Ediciones Universitarias de Valparaíso, Pontificia Universidad Católica de Valparaíso.

Vespucci, A. (1985.). Lettrere de Viaggio a cura di Luiciano Formisano. Mondadori, Milano.

Vespucci, A. (2005). Le Nouveau Monde. Les voyages d'Amerigo Vespucci (1497-1504). Paris: Chandeigne.

White, T. (1976). Aristotle and Utopia. Renaissance Quarterly, 29 (4), 635-675.

Zweig, S. (2010). Américo Vespucio. Madrid: Capitan Swing. 
